\title{
COVID-19: What lodine Maps From Perfusion CT can reveal-A Prospective Cohort Study
}

\author{
Mario G. Santamarina ${ }^{1,2^{*}} \mathbb{D}$, Dominique Boisier Riscal ${ }^{3}$, Ignacio Beddings ${ }^{4}$, Roberto Contreras ${ }^{5}$, \\ Martiniano Baque ${ }^{6}$, Mariano Volpacchio ${ }^{7}$ and Felipe Martinez Lomakin ${ }^{8,3}$
}

\begin{abstract}
Background: Subtraction CT angiography (SCTA) is a technique used to evaluate pulmonary perfusion based on iodine distribution maps. The aim of this study is to assess lung perfusion changes with sCTA seen in patients with COVID-19 pneumonia and correlate them with clinical outcomes.

Material and methods: A prospective cohort study was carried out with 45 RT-PCR-confirmed COVID-19 patients that required hospitalization at three different hospitals, between April and May 2020. In all cases, a basic clinical and demographic profile was obtained. Lung perfusion was assessed using sCTA. Evaluated imaging features included: Pattern predominance of injured lung parenchyma in both lungs (ground-glass opacities, consolidation and mixed pattern) and anatomical extension; predominant type of perfusion abnormality (increased perfusion or hypoperfusion), perfusion abnormality distribution (focal or diffuse), extension of perfusion abnormalities (mild, moderate and severe involvement); presence of vascular dilatation and vascular tortuosity. All participants were followed-up until hospital discharge searching for the development of any of the study endpoints. These endpoints included intensivecare unit (ICU) admission, initiation of invasive mechanical ventilation (IMV) and death.
\end{abstract}

Results: Forty-one patients (55.2 \pm 16.5 years, 22 men) with RT-PCR-confirmed SARS-CoV-2 infection and an interpretable iodine map were included. Patients with perfusion anomalies on SCTA in morphologically normal lung parenchyma showed lower Pa/Fi values (294 \pm 111.3 vs. $397 \pm 37.7, p=0.035)$, and higher D-dimer levels (1156 \pm 1018 vs. $378 \pm 60.2, p<0.01)$. The main common patterns seen in lung CT scans were ground-glass opacities, mixed pattern with predominant ground-glass opacities and mixed pattern with predominant consolidation in $56.1 \%, 24.4 \%$ and $19.5 \%$ respectively. Perfusion abnormalities were common (36 patients, $87.8 \%$ ), mainly hypoperfusion in areas of apparently healthy lung. Patients with severe hypoperfusion in areas of apparently healthy lung parenchyma had an increased probability of being admitted to ICU and to initiate IMV (HR of $11.9(95 \% \mathrm{Cl} 1.55-91.9)$ and $\mathrm{HR} 7.8(95 \% \mathrm{Cl}$ 1.05-61.1), respectively).

Conclusion: Perfusion abnormalities evidenced in iodine maps obtained by SCTA are associated with increased admission to ICU and initiation of IMV in COVID-19 patients.

Keywords: COVID-19, Coronavirus, Computed tomography angiography, Angiotensin converting enzyme 2, Angiotensin II, Vasoconstriction, Vasoplegia, Ventilation-perfusion ratio

*Correspondence: mgsantama@yahoo.com

${ }^{1}$ Radiology Department, Hospital Naval Almirante Nef, Subida Alesandri S/N., Viña del Mar, Provincia de Valparaíso, Chile

Full list of author information is available at the end of the article

\section{Background}

In December 2019, a novel human coronavirus, SARSCoV-2, was reported in the city of Wuhan, China. It has spread throughout the world and caused a pandemic coronavirus disease (COVID-19). As of 22th July 2020, the disease had affected more than 15 million people causing original author(s) and the source, provide a link to the Creative Commons licence, and indicate if changes were made. The images or other third party material in this article are included in the article's Creative Commons licence, unless indicated otherwise in a credit line to the material. If material is not included in the article's Creative Commons licence and your intended use is not permitted by statutory regulation or exceeds the permitted use, you will need to obtain permission directly from the copyright holder. To view a copy of this licence, visit http://creativecommons.org/licenses/by/4.0/. The Creative Commons Public Domain Dedication waiver (http://creativeco mmons.org/publicdomain/zero/1.0/) applies to the data made available in this article, unless otherwise stated in a credit line to the data. 
more than 620,000 deaths, with the United States becoming the latest epicenter with near 3,960,000 cases and the highest number of deaths-over 140,000 people [1].

SARS-CoV-2 infection begins with inoculation of the respiratory tract mucosa using angiotensin-converting enzyme 2 (ACE2) as the functional receptor for cell entry. The inhaled virus likely binds to epithelial cells in the nasal cavity and starts replicating. There is local propagation of the virus, with a limited initial innate immune response. At this stage the virus can be detected via nasal swabs, and although viral burden may be low, these individuals are contagious. The virus propagates and migrates down the respiratory tract along the conducting airways, and a more robust innate immune response is triggered. For about $80 \%$ of infected patients, the disease will be mild and mostly restricted to the upper and conducting airways. However, the remaining $20 \%$ of infected patients will progress and have severe pulmonary infiltrates, while some of these will develop very severe disease and eventually die [2].

A wealth of scientific data has been published over the past few months on epidemiology, clinical manifestations, molecular biology, imaging features, laboratory diagnosis and treatments, but evidence has not been robust in most literature. Although COVID-19 pneumonia may sometimes meet the ARDS Berlin definition criteria, some authors argue that it is a specific disease with peculiar phenotypes, due to the frequent dissociation between the severity of hypoxemia and the maintenance of relatively good respiratory mechanics [3].

Imaging plays a crucial role in the initial detection and evaluation of progression of COVID-19 pneumonia.

To the best of our knowledge, there is currently little scientific data regarding lung perfusion disturbances occurring in patients with COVID-19 pneumonia [4]. Our goal is to assess lung perfusion changes seen in patients with COVID-19 pneumonia, and to correlate perfusion abnormalities with clinical outcomes.

\section{Materials and methods Patient cohort}

This study was approved by our Institutional Review Board at the Hospital Naval Almirante Nef and written informed consent was waived. A prospective cohort study was undertaken to assess the prognostic ability of lung perfusion using subtraction CT angiography (sCTA). Patients ( $>18$ years old) with confirmed SARS-CoV-2 infection by real-time reverse transcription polymerase chain reaction (RT-PCR) that required hospitalization were consecutively enrolled at three hospitals between April and May 2020. These centers included the Hospital Naval Almirante Nef (Viña del Mar, Chile), Hospital San Martín de Quillota (Quillota, Chile), and
Hospital IESS Los Ceibos (Guayaquil, Ecuador). These facilities are tertiary-care hospitals able to provide onsite CTA scans and intensive care management of severe COVID-19 cases when needed.

Forty-five patients were included in this study. However, due to uninterpretable iodine map results, four of these participants had to be excluded from analyses. This left 41 patients (22 males and 19 females) in the study. In all cases, a basic clinical and demographic profile was obtained that included information regarding sex, age, duration of clinical symptoms, and laboratory studies including $\mathrm{D}$-dimer, alveolar oxygenation to inspired oxygen $(\mathrm{Pa} / \mathrm{Fi})$ ratio at baseline and sequential organ failure assessment (SOFA) score. Baseline lung CT characteristics were recorded as well such as the predominant imaging pattern (ground-glass opacities, consolidation or mixed pattern), the extension of involved lung, presence of pleural effusion, evidence of right-ventricular overload and pulmonary embolism. These data were recorded along with sCTA features (see below) in an anonymized registry.

All participants were followed-up until hospital discharge searching for the development of any of the study endpoints. These endpoints included intensive-care unit (ICU) admission, initiation of invasive mechanical ventilation (IMV) and death. The decision to intubate or admit to an ICU facility was left to the attending physician's discretion.

\section{CT scan protocol and image acquisition}

All CT scans were performed within $24 \mathrm{~h}$ of admission to the hospital, in supine position. Imaging data were acquired with multidetector-CT (Canon Aquilion Prime 160 and 80, and Canon Aquilion RXL 16).

\section{CT scan protocol}

After positioning, an unenhanced scan was obtained, followed by IV injection of $100 \mathrm{~mL}$ iodinated contrast medium at a rate of $5 \mathrm{~mL} / \mathrm{seg}$ (Visipaque 320, GE Healthcare, Milwaukee, Wis and Optiray 320, Mallinckrodt Medical, St Louis, Mo). After bolus triggering at the level of the pulmonary artery with a relative threshold of 150 $\mathrm{HU}$, an early pulmonary arterial angiographic phase was obtained, followed eight seconds later by a delayed pulmonary arterial phase. All the exams were performed with the same acquisition parameters: $\mathrm{kV}(100 \mathrm{kV})$, automatic exposure control (Standard), similar range and field-of-view (FOV: L or LL), collimation $(1.0 \times 16$ for Canon Aquilion RXL 16; $0.5 \times 80$ for Canon Aquilion Prime 80 and 160), pitch factor (1.4), and same rotation speed $(0.5 \mathrm{~s}$ for Canon Aquilion RXL 16 and $0.35 \mathrm{~s}$ for Canon Aquilion Prime 80 and 160). The acquired images had the same reconstruction parameters: slice thickness 
$(1.0 \mathrm{~mm})$, reconstruction interval $(0.8 \mathrm{~mm})$ and convolution filter (mediastinum). Average dose DLP (mGy/cm) was 1093 (range: 847-1932). Breathing instructions were the same for all examinations.

sCTA is a technique that uses software-based motion correction between an unenhanced and an enhanced CT scan to obtain an iodine distribution map of the lung parenchyma. Iodine distribution maps of the early and delayed arterial phases were obtained using SureSubtraction software (version 7.0; Canon Medical Systems, Japan). Iodine maps were generated with grayscale and identical color window tables, ranging from blue (low iodine enhancement) to yellow (high iodine enhancement).

\section{Image analysis}

Areas of injured parenchyma in both lungs were assessed for a predominant pattern. These were characterized as ground glass opacities, consolidation and mixed pattern. Airspace compromise was assessed for each of the 5 lobes considering the extent of anatomic involvement, as follows: 0 points for no involvement; 1 point for $<30 \%$ involvement; 2 points for $31-60 \%$ involvement; and 3 points for $>61 \%$ involvement. The resulting CT severity score was the sum of each individual lobar score (0-15). Areas of airspace disease were also assessed for the presence of increased perfusion or hypoperfusion. Peripheral vascular dilatation and vascular tortuosity were also assessed in each lobe.

Areas of apparently healthy lung parenchyma were assessed for preservation of anteroposterior and apicobasal perfusion gradient or hypoperfusion. Hypoperfusion in apparently normal lung parenchyma was characterized according to its distribution (focal or diffuse), and extension. The latter was graded using a scoring system: first, both lungs were divided into five lobes (upper right and left lobes, middle lobe, and lower right and left lobes). Then, the extension of hypoperfusion in apparently healthy lung parenchyma in each lobe was categorized as normal perfusion ( 0 points), less than $50 \%$ of the lobe affected (1 point), and $50 \%$ of the lobe or more affected ( 2 points). This resulted in an overall score that ranged from 0 to 10 points, with higher scores indicating more severe hypoperfusion. Using this score, patients were divided in three groups. Patients with 3 points or less were considered to have mild perfusion abnormalities, those with 4-6 points had moderate abnormalities and those with 7 or more points had severe abnormalities.

A single radiologist with over 15-years experience (MS) in reading $\mathrm{CT}$ analyzed the images of included patients in all hospitals. The attending physicians did not have information regarding sCTA perfusion results. However, data regarding overall $\mathrm{CT}$ characteristics of included participants was made available to clinicians as per current protocols at study centers.

\section{Statistical analyses}

Descriptive statistics including medians, means, standard deviations, interquartile ranges (IQR) and absolute and relative frequencies were used first to describe the study sample. Bivariate comparisons between groups were performed using Fisher's Exact test for categorical variables and Student's t test or Mann-Whitney's test for continuous variables after reviewing data distribution and variances. Association between quantitative variables were sought using Pearson's or Kendall's correlation coefficients. Discrete quantitative variables (such as computed tomography scores) were evaluated using Kendall's tau coefficient. The development of any of the aforementioned study endpoints was evaluated using KaplanMeier survival curves which were then compared using the logrank statistic. Ninety-five percent confidence intervals were calculated whenever appropriate. Analyses were undertaken by an independent statistician who had no participation in the decision to admit or intubate any of the included patients, using STATA $15.0 \mathrm{SE}^{\circledR}$ (College Station, TX: StataCorp LLC). A two-sided $p$ value of $<5 \%$ was considered to be statistically significant.

\section{Results}

\section{Patient characteristics}

All patients had SARS-CoV-2 infection confirmed by a positive RT-PCR. The mean age was $55.2 \pm 16.5$ years and the median duration of symptoms was 7 (IQR 5-8) days. Laboratory investigations were often abnormal. Twentythree patients (59\%) had abnormal D-dimer levels, which averaged $1057 \pm 984.1 \mathrm{ng} / \mathrm{mL}$. The average $\mathrm{Pa} / \mathrm{Fi}$ ratio on admission was $307 \pm 110.2$ and the median SOFA score was 2 (IQR 1-2) points, which was similar between study groups $(p=0.09)$. Patients with hypoperfusion abnormalities on apparently healthy lung parenchyma on sCTA showed lower $\mathrm{Pa} / \mathrm{Fi}$ values $(294 \pm 111.3$ vs. $397 \pm 37.7$, $p=0.035)$, and higher $\mathrm{D}$-dimer levels $(1156 \pm 1018$ vs. $378 \pm 60.2, p<0.01)$. A summary of baseline clinical characteristics is shown in Table 1.

\section{Lung $\mathrm{CT}$ evaluation}

The most common patterns seen in lung CT scans were ground-glass opacities (23 patients, 56.1\%), followed by mixed pattern with predominant ground-glass opacities (10 patients, $24.4 \%$ ) and mixed pattern with predominant consolidation (8 patients, $19.5 \%$ ).

There were no major differences in the predominant radiological pattern between normal and abnormal perfusion groups. Multilobar lung involvement was common among included patients but tended to be less among 
Table 1 Baseline characteristics

\begin{tabular}{|c|c|c|c|c|}
\hline Characteristic & $\begin{array}{l}\text { Normal perfusion } C T \\
(n=5)\end{array}$ & $\begin{array}{l}\text { Abnormal perfusion } C T \\
(n=36)\end{array}$ & Total $(n=41)$ & $p$ value \\
\hline \multicolumn{5}{|l|}{ Clinical characteristics } \\
\hline Mean age (years) (SD) & $46.8 \pm 11.5$ & $56.4 \pm 16.8$ & $55.2 \pm 16.5$ & $0.28^{\mathrm{a}}$ \\
\hline Male sex $(n, \%)$ & $1(20 \%)$ & $21(58.3 \%)$ & $22(53.7 \%)$ & $0.16^{b}$ \\
\hline Median SOFA score (IQR) & $1(1)$ & $2(1-3)$ & $2(1-2)$ & $0.09^{b}$ \\
\hline Median duration of symptoms (IQR) & $6(4-7)$ & $7(5-8)$ & $7(5-8)$ & $0.44^{\mathrm{a}}$ \\
\hline Mean $\mathrm{Pa} /$ Fi ratio (SD) & $397 \pm 37.7$ & $294 \pm 111.3$ & $307 \pm 110.2$ & $0.035^{\mathrm{a}}$ \\
\hline Mean D-Dimer levels (ng/mL) (SD) & $378 \pm 60.2$ & $1156 \pm 1018.1$ & $1057 \pm 984.1$ & $<0.01$ \\
\hline \multicolumn{5}{|l|}{ Computed tomography findings } \\
\hline \multicolumn{5}{|l|}{ Predominant pattern (n, \%) } \\
\hline Ground-glass opacities & $5(100 \%)$ & $18(50 \%)$ & $23(56.1 \%)$ & $0.21^{b}$ \\
\hline Mixed pattern with predominant ground-glass opacities & $0(0 \%)$ & $10(27.8 \%)$ & $10(24.4 \%)$ & \\
\hline Mixed pattern with predominant consolidation & $0(0 \%)$ & $8(22.2 \%)$ & $8(19.5 \%)$ & \\
\hline Median number of compromised lobes (IQR) & $4(2-5)$ & $5(5)$ & $5(5)$ & $0.02^{\mathrm{a}}$ \\
\hline Pleural effusion ( $n, \%)$ & $2(40 \%)$ & $5(13.9 \%)$ & $7(17.1 \%)$ & $0.20^{b}$ \\
\hline Pulmonary embolism (n, \%) & $0(0 \%)$ & $2(5.6 \%)$ & $2(4.8 \%)$ & $1.0^{b}$ \\
\hline Right ventricular overload (n, \%) & $0(0 \%)$ & $0(0 \%)$ & $0(0 \%)$ & - \\
\hline Vascular dilatation (n, \%) & $4(80 \%)$ & $36(100 \%)$ & $40(97.5 \%)$ & $0.12^{b}$ \\
\hline Median number of lobes with vascular dilatation (IQR) & $2(2)$ & $3.5(2-5)$ & $3(2-5)$ & $0.17^{\mathrm{a}}$ \\
\hline Vascular tortuosity (n, \%) & $0(0 \%)$ & $14(38.9 \%)$ & $14(34.2 \%)$ & $0.15^{b}$ \\
\hline Median CT severity score (IQR) & $3(2-5)$ & $7(5-10)$ & $7(5-10)$ & $0.01^{b}$ \\
\hline
\end{tabular}

patients without perfusion abnormalities $(p=0.02)$. In the same way, the median CT severity score was higher and statistically significant in the group with perfusion abnormalities (7 (IQR 5-10) vs. 3 (IQR 2-5), $p=0.01$ ).

Pulmonary embolism was found in two patients (4.8\%), and both had an abnormal perfusion CT. Pleural effusion was infrequent among included participants (7 patients, $17.1 \%)$. No patient showed signs of right ventricular overload at baseline. Vascular dilatation, defined as a 1.5-fold increase in arterial diameter compared to the accompanying bronchus, was observed in 40 (97.5\%) patients. Vascular tortuosity was found in 14 patients, all belonging to the group with perfusion abnormalities. The median number of lobes with vascular dilatation was also higher in the group that showed perfusion anomalies. The presence of vascular tortuosity was significantly associated with the requirement of invasive mechanical ventilation (HR 5.9, 95\% CI 1.83-18.91, $p=0.003$ ) (Fig. 1).

\section{Perfusion findings and study outcomes}

Perfusion abnormalities were common among included participants with $36(87.8 \%)$ patients having hypoperfusion in areas of non-injured parenchyma. These anomalies had most commonly a diffuse distribution (23 patients, $63.9 \%$ ). The median perfusion-CT score for areas of decreased perfusion was 5 points (IQR 3-9). Patients were then categorized in three groups using the aforementioned scoring system according to the extension of perfusion abnormalities in non-injured lung. Eleven patients $(26.8 \%)$ were identified as having mild perfusion anomalies, 13 patients $(31.7 \%)$ had moderate perfusion abnormalities and $17(41.5 \%)$ had severe abnormalities (Figs. 2, 3).

Nineteen patients $(46.3 \%)$ were admitted to the ICU during follow up, and 14 (34.2\%) required IMV. Two patients $(4.9 \%)$ died during hospitalization. None of the patients with normal perfusion CT scans required admission to the ICU or IMV, and all survived. Survival analyses showed significant differences in the probability of ICU admission and initiation of IMV between perfusion score groups, which are shown in Fig. 4a, b. Patients with severe hypoperfusion in apparently healthy lung parenchyma had a greatly increased probability of being admitted to the ICU, with a HR of 11.9 (95\% CI 1.55-91.9, $p=0.017$ ) when contrasted with patients with mild perfusion abnormalities. Similar findings were seen regarding the initiation of IMV (HR 


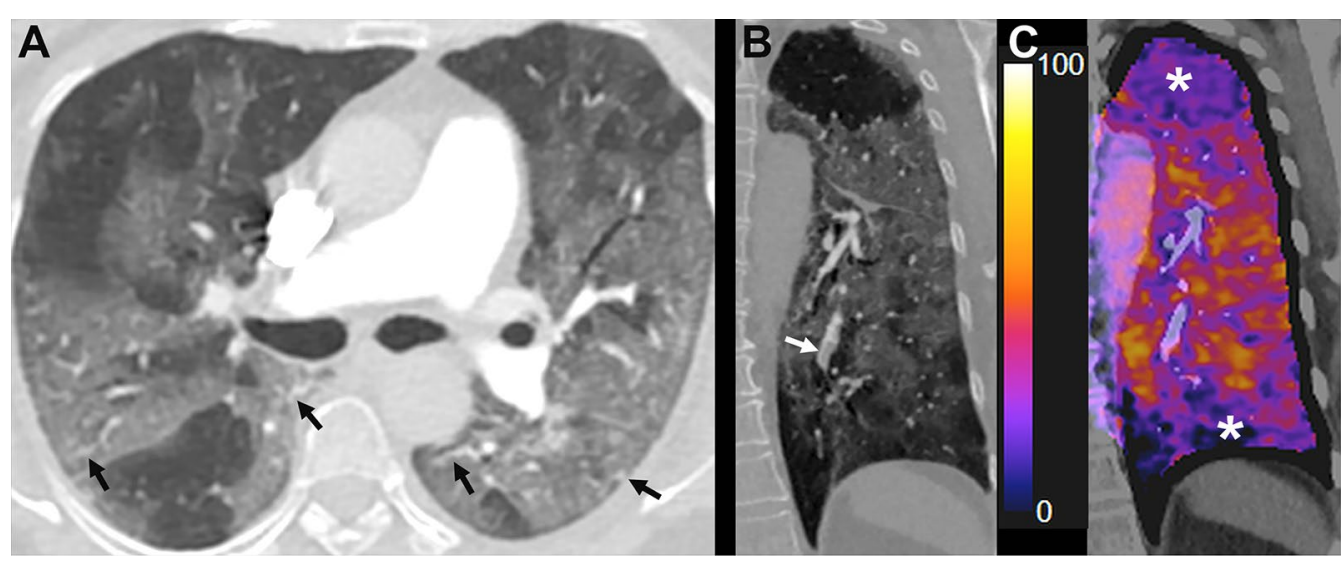

Fig. 1. 78-year-old female patient, RT-PCR-confirmed COVID-19, 8 days since symptom onset, complicated with pulmonary embolism. Admission $\mathrm{PaO}_{2} / \mathrm{FiO}_{2}$ ratio was 162 , and d-dimer levels $>2000 \mathrm{ng} / \mathrm{mL}$. Admitted to the intensive care unit, managed with invasive mechanical ventilation. She died 2 weeks after admission. a Axial lung-window CT angiography image shows extensive lung involvement with patchy ground-glass opacities in both lungs, with vascular dilatation in small peripheral subsegmental pulmonary arterial branches, some of them with a varicose appearance (black arrows). $\mathbf{b}$ Coronal CT angiography image shows pulmonary embolism in the posterior basal segment of the lower left lobe (white arrow). $5 \mathrm{~mm}$ coronal-plane reconstruction of a subtraction iodine map shows moderate to severe hypoperfusion in superior and inferior regions of the lung $\left(^{*}\right)$, in areas of apparently healthy lung parenchyma in conventional chest CT images, which is more pronounced in the posterior basal segment of the lower left lobe, in relation to the area of pulmonary embolism. Areas of ground-glass opacities show normal or increased perfusion, most probably due to vasoplegia

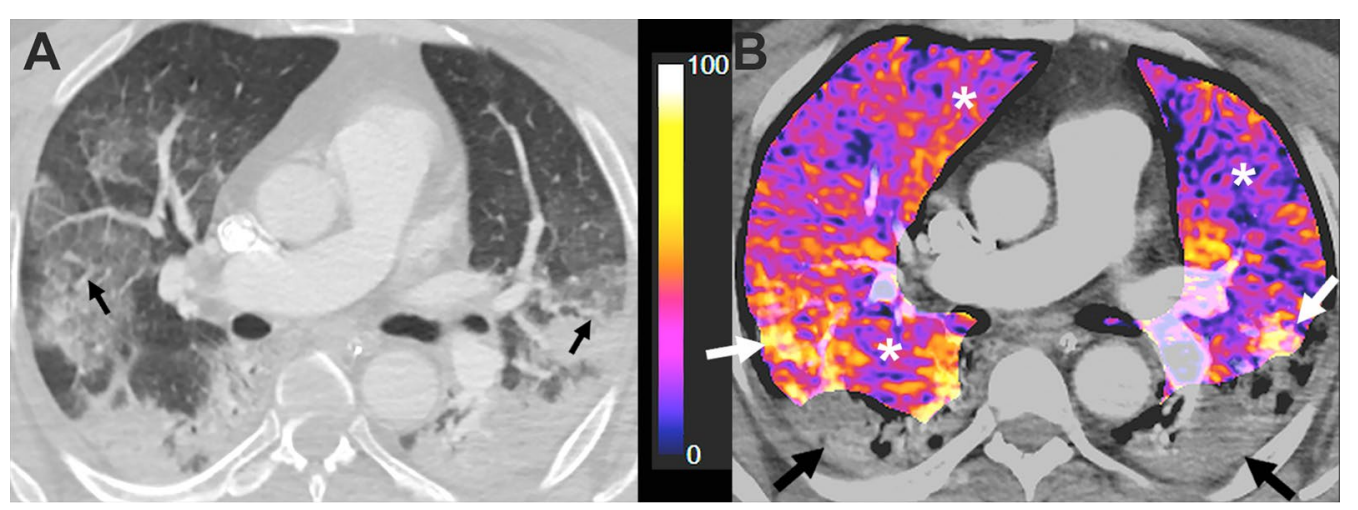

Fig. 2. 51-year-old male patient, RT-PCR confirmed COVID-19, 3 days since symptom onset. Admission $\mathrm{PaO}_{2} / \mathrm{FiO}_{2}$ ratio was 240 , and d-dimer level was $480 \mathrm{ng} / \mathrm{mL}$. Admitted to the intensive care unit, managed with invasive mechanical ventilation. a Axial lung-window CT angiography image shows extensive bilateral ground-glass opacities, with areas of posterior subpleural consolidation. There is subtle subsegmental peripheral vascular dilatation of pulmonary arterial branches (small black arrows). b $5 \mathrm{~mm}$ axial reconstruction of a subtraction iodine map shows slight to moderate hypoperfusion predominantly in areas of non-injured lung $\left({ }^{*}\right)$, and more prominent areas of increased perfusion in relation to the zones of ground-glass opacities (white arrows). Perfusion was not assessed in the areas of consolidation (black arrows) due to software limitations that exclude these zones. This patient evolved in a similar fashion to the $\mathrm{H}$ (type 2) phenotype described by Gattinoni et al.

7.8 95\% CI $1.05-61.1, p=0.025)$. Patients with moderate hypoperfusion anomalies also had an increased risk of ICU admission and of requiring IMV, albeit without statistical significance (HR 4.5 95\% CI 0.5338.4, $p=0.17$ and HR 2.60, 95\% CI $0.27-25, p=0.41$, respectively). A summary of study outcomes is shown in Table 2. Thirty-four of these patients (94.4\%) showed increased perfusion to areas with visible parenchymal abnormalities, with the remaining two patients showing perfusion defects in these zones (5.6\%) as their predominant finding (Fig. 5). 14 (34\%) patients had mild artifacts on iodine maps that did not interfere with the analysis of the images. A moderate correlation was found between perfusion $\mathrm{CT}$ score and CT severity score $(\mathrm{TauB}=0.47)$, which was statistically significant $(p<0.001)$. This association is shown in Fig. 6. 


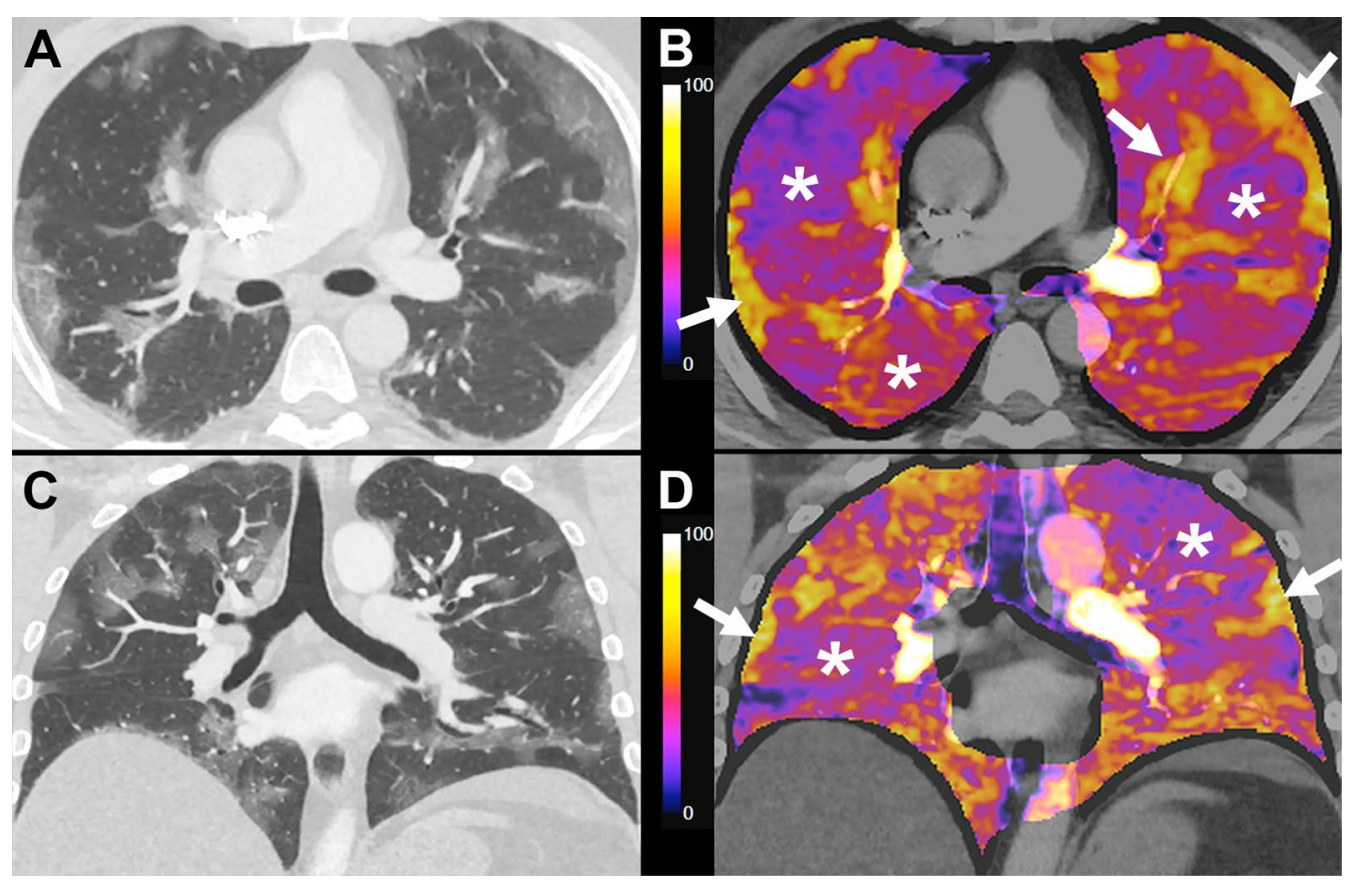

Fig. 3. 37 -year-old male patient, RT-PCR-confirmed COVID-19, 10 days since symptom onset. Admission $\mathrm{PaO}_{2} / \mathrm{FiO}_{2}$ ratio was 240 and d-dimer level was $480 \mathrm{ng} / \mathrm{mL}$. Admitted to the intensive care unit, managed with invasive mechanical ventilation. a, c Axial and coronal lung-window chest CT angiography images show multiple foci of ground-glass opacities, with a predominantly subpleural distribution, with areas of apparently healthy lung parenchyma. Vascular dilatation can be seen in relation to areas of ground-glass opacities. $\mathbf{b}, \mathbf{d} 5 \mathrm{~mm}$ reconstruction images of subtraction iodine maps in corresponding axial and coronal planes, show areas of severe hypoperfusion in the corresponding apparently healthy lung parenchyma $(*)$, with increased perfusion in areas of ground-glass opacities (white arrows). This patient evolved in a similar fashion to the $L$ (type 1) phenotype described by Gattinoni et al.
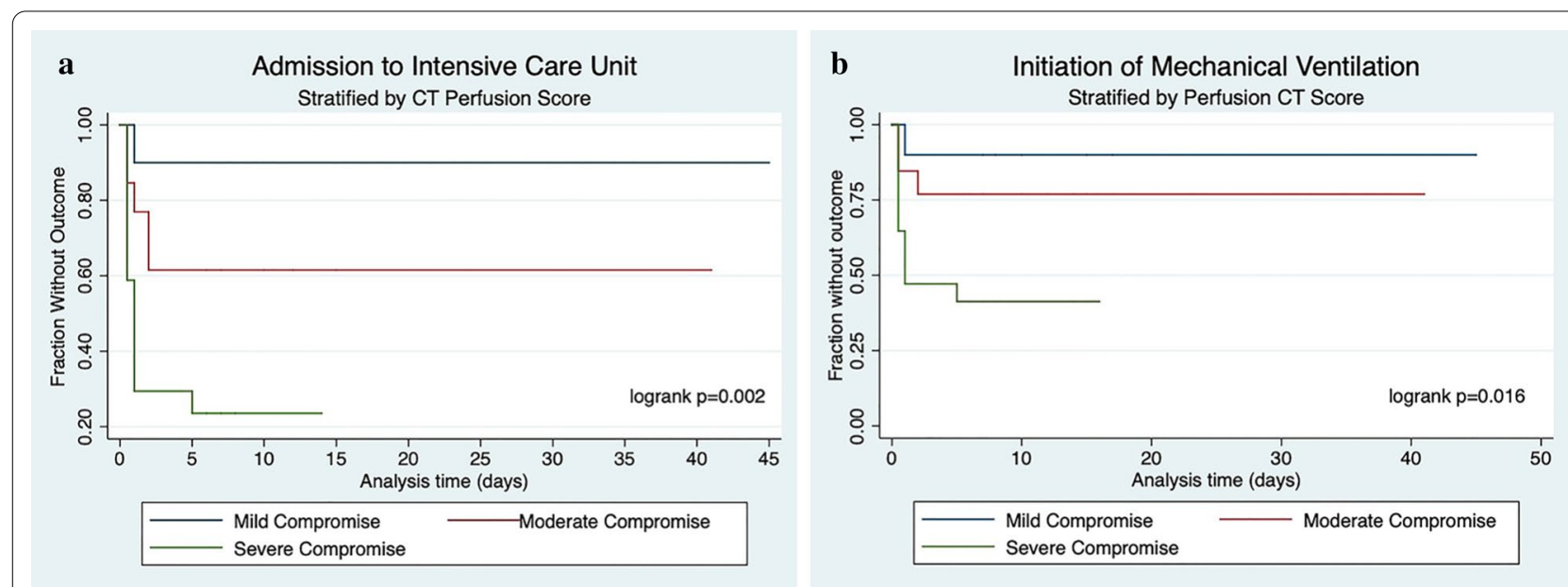

Fig. 4 a Admission to Intensive Care Unit. Stratified by CT Perfusion Score. b Initiation of Mechanical Ventilation. Stratified by CT Perfusion Score

\section{Discussion}

With the advent of SARS-Cov-2, nearly $20 \%$ of the infected patients will progress and develop severe disease, consistent in acute hypoxemic respiratory failure, and eventually die [2]. In our prospective cohort, we evaluated and quantified lung perfusion disturbances in patients with COVID-19 pneumonia as a severity predictor, through iodine distribution maps obtained with sCTA. Our findings showed that with increasing severity of hypoperfusion abnormalities in areas of apparently 
Table 2 Study outcomes

\begin{tabular}{|c|c|c|c|c|}
\hline Outcome & $\begin{array}{l}\text { Mild } \\
\text { compromise } \\
(n=11)\end{array}$ & $\begin{array}{l}\text { Moderate } \\
\text { compromise } \\
(n=13)\end{array}$ & $\begin{array}{l}\text { Severe } \\
\text { compromise } \\
(n=17)\end{array}$ & $p$ value $^{a}$ \\
\hline $\begin{array}{l}\text { Admission to } \\
\text { intensive } \\
\text { care unit } \\
(n, \%)\end{array}$ & $1(9.1 \%)$ & $5(38.5 \%)$ & 13 (76.5\%) & 0.002 \\
\hline $\begin{array}{l}\text { Initiation of } \\
\text { mechanical } \\
\text { ventilation } \\
(n, \%)\end{array}$ & 1 (11.1\%) & $3(23.1 \%)$ & $10(58.8 \%)$ & 0.016 \\
\hline Death (n, \%) & $0(0 \%)$ & $1(7.7 \%)$ & $1(5.9 \%)$ & 0.72 \\
\hline
\end{tabular}

healthy lung parenchyma in conventional chest CT images, patients had a statistically significant increase in their chance to require admission to ICU $(p=0.002)$, and to require IMV $(p=0.016)$. Vascular tortuosity in CT angiography images was also independently associated with requirement of IMV $(p=0.003)$. We also found that average lower $\mathrm{Pa} / \mathrm{Fi}$ ratio $(p=0.035)$, higher $\mathrm{D}$-dimer levels $(p<0.01)$, and number of affected lung lobules $(p=0.02)$, were associated with perfusion abnormalities.

Patients with COVID-19 pneumonia showed abnormally decreased iodine distribution in areas of apparently normal lung parenchyma, findings that as a whole probably account for a severe V/Q mismatch. Some authors have suggested microthrombi to be a key component of clinical deterioration. Activation of the coagulation cascade in early stages of disease appears to be due to a thromboinflammatory response and direct viral effects on pulmonary tissue, which is counterbalanced by fibrinolytic activity and manifests as an increase in fibrin degradation products. In later stages of disease, this balance is lost and a pro-coagulant state ensues [5-8].

ACE2 dysfunction could be a possible explanation for the establishment of a systemic endotheliopathy that may lead to an abnormal pro-coagulant state and sepsis [9]. We believe that the main pathophysiologic mechanism behind COVID-19 pneumonia involves accumulation of angiotensin II due to decreased activity of ACE2, which is secondary to ACE2 endocytosis after viral binding [10]. This leads to vasoconstriction, establishing a progressive V/Q mismatch, with extensive areas of apparently healthy but hypoperfused lung that function as alveolar dead space.

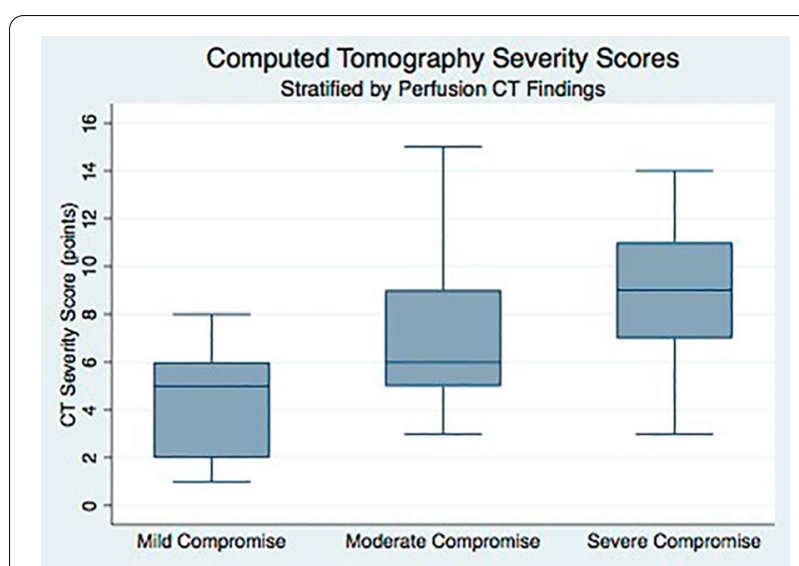

Fig. 6 Computed Tomography Severity Scores stratified by Perfusion CT Findings

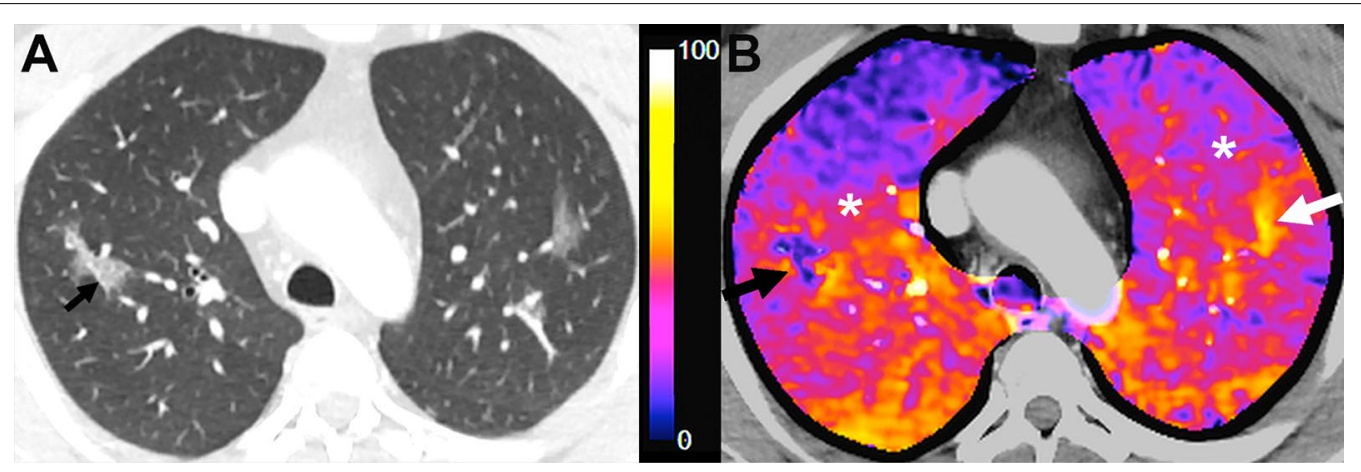

Fig. 5. 40-year-old male patient, RT-PCR-confirmed COVID-19, 5 days since symptom onset. Admission $\mathrm{PaO}_{2} / \mathrm{FiO}_{2}$ ratio was 310 , and d-dimer levels $<300 \mathrm{ng} / \mathrm{mL}$. Due to progressive hypoxaemia, he was managed in the intensive care unit with conscious prone position and high flow nasal cannula, with positive tolerance and evolution. a Axial lung-window CT angiography image shows small patchy areas of ground-glass opacities, with slightly dilated small pulmonary arterial branches (small black arrow). b $5 \mathrm{~mm}$ axial reconstruction of a subtraction iodine map shows moderate hypoperfusion with a right-sided predominance $\left(^{*}\right)$. The ground-glass opacity in the upper right lobe shows decreased perfusion within the opacity, with a peripheral halo of increased perfusion (black arrow). These findings could be explained by physiological hypoxic vasoconstriction. However, the ground-glass opacity in the upper left lobe shows increased perfusion (white arrow) 
This is supported by studies showing evidence that non-hypertense patients with COVID-19 appeared to have elevated levels of plasma angiotensin II, which were correlated with the degree of lung injury and total viral load [11, 12].

Decreased activity of ACE2 leads to heightened and relatively unopposed vasoconstriction, pro-coagulation, pro-inflammatory and pro-oxidant angiotensin II effects $[13,14]$.

In a scenario of extensive vasoconstriction in well-ventilated areas, possible treatment strategies could be aimed at improving perfusion. Luni Chen et al. treated patients with severe SARS in 2003 with inhaled nitric oxide, with significant improvement in arterial oxygenation [15].

Areas of injured lung parenchyma characterized by ground-glass opacities, consolidation and septal thickening, also have particular anomalies in COVID-19 pneumonia. Subsegmental vessel enlargement in the vicinity of areas of injured parenchyma has been described in up to $89 \%$ of patients with COVID-19 pneumonia [2]. Although in situ microthrombosis is a possible underlying mechanism, it could also be explained by vasoplegia induced by SARS-CoV-2. Overactivation of a regional vasodilatation cascade in areas of injured non-aerated lung parenchyma, due to a local dysfunctional inflammatory response, could explain the loss of the normal, physiological hypoxic arterial vasoconstriction that would normally be expected in areas of hypoventilated lung, contributing to the abnormal hyperperfusion that we have found in areas of lung opacities.

Gattinoni et al. [3] hypothesized that there are two distinct types of lung damage in ARDS. Type-L or 1 is characterized by low elastance and high compliance, which is rarely seen in ARDS, while type- $\mathrm{H}$ or 2 shows high elastance and low compliance that is typical of ARDS. They explain the primary cause of hypoxemia in type- $\mathrm{L}$ is perfusion defects presumably caused by vasoconstriction and high shunt fraction. In contrast, the high elastance in type- $\mathrm{H}$ is thought to be induced by lung edema.

In type-L pattern, areas of injured lung parenchyma show hyperperfusion, probably due to loss of compensatory hypoxic pulmonary vasoconstriction (vasoplegia), resulting in high perfusion to areas of hypoventilated lung and an abnormally low V/Q ratio; while areas of apparently normal lung parenchyma show hypoperfusion that results in an abnormally high V/Q ratio. Changes in vascular resistance lead to a net shunt or steal of vascular flow towards areas of non-aerated lung, which is supported by the good clinical response to prone position that has been described in patients with COVID-19 [16].

This study has several limitations. First, the small sample size, mainly explained by the epidemiologic situation of the cities where the participant hospitals are located at the moment of the study, which were in early stages of the pandemic outbreak. Our relatively small sample size restricted the use of multivariable techniques to further refine the association with perfusion $\mathrm{CT}$ findings and prognosis. Although the bivariate contrasts were both clinically and statistically significant, it is not possible to exclude bias due to confounding, especially considering the correlation shown in Fig. 6. However, it should also be kept in mind that the overall strength of the correlation was only moderate. This reduces the possibility that our findings are explained solely by confounding. Further studies with larger sample sizes are needed to fully assess this association.

Second, the analysis of the images was performed by a single radiologist, resulting in a lack of inter-observer variability assessment. Third, perfusion imaging involves more radiation than conventional $\mathrm{CT}$ techniques. Fourth, dual-energy CT (DECT) is commonly used in clinical practice for assessing pulmonary perfusion and uses material decomposition of iodine from other materials to visualize the regional distribution of intravenous contrast in the pulmonary vessels, including the capillaries [17, 18]. Although SCTA is a new postprocessing technique and thus, has not been as extensively validated as DECT, it is a promising approach that digitally subtracts a precontrast CT scan from a contrast material-enhanced CT scan after motion correction. Since it is software-based, it is significantly less expensive and potentially more available than costly DECT equipment. Nevertheless, being a novel approach, concerns regarding potential bias resulting from subtraction of non-contrast images from contrast-enhanced images need to be addressed, and greater experience and better knowledge of typical pitfalls is needed to improve the diagnostic accuracy [19-21]. Fifth, some of the iodine maps were uninterpretable. Finally, it is unclear whether these perfusion abnormalities are unique to COVID-19 or if they can also be found in other multifocal pneumonias and other causes of ARDS.

\section{Conclusions}

Severe lung perfusion abnormalities, evaluated by iodine maps, were associated with a higher ICU admission and initiation of IMV. Perfusion abnormalities were detected in both damaged and normal lung parenchyma, allowing us to state that different types of V/Q mismatch occur in the entire lung parenchyma in COVID-19 patients. These findings suggest considering the evaluation of therapies that are oriented towards improving pulmonary perfusion in COVID-19 and maybe could contribute to the management and outcome of these patients. The prognostic implications of hypoperfusion in normal lung 
parenchyma remain to be assessed independently from severity and extension of lung opacities.

\author{
Abbreviations \\ COVID-19: Coronavirus Disease 2019; ACE2: Angiotensin-converting enzyme \\ 2; sCTA: Subtraction CT angiography; ICU: Intensive Care Unit; IMV: Invasive \\ mechanical ventilation.
}

\section{Acknowledgements}

Not applicable.

\section{Summary statement}

Lung perfusion abnormalities in patients with COVID-19 pneumonia were associated with admission to Intensive Care Unit and requirement of invasive mechanical ventilation.

\section{Authors' contributions}

MS: Clinical question design, image acquisition protocolization, image interpretation and analysis, literature review, co-wrote the paper. DBR: Clinical question design, literature review, co-wrote the paper. IB: Clinical question design, image interpretation and analysis, literature review, co-wrote the paper. RC: Helped in identifying the clinical question, provided clinical input, helped to draft the paper. MB: Provided clinical input, helped to draft the paper. MV: Helped with image acquisition protocolization, helped with drafting and revision of the paper. FML: Study design, statistical analysis, co-wrote the paper. The authors read and approved the final manuscript.

\section{Funding}

This work did not receive public or private funding.

\section{Availability of data and materials}

The datasets used and/or analyzed during the current study are available from the corresponding author on reasonable request.

\section{Ethics approval and consent to participate}

This study was approved by the Institutional Review Board at the Hospital Naval Almirante Nef. The requirement to obtain informed consent from patients enrolled in the aforementioned study was waived.

\section{Consent for publication}

Not applicable.

\section{Competing interests}

The authors declare that they have no competing interests.

\section{Author details}

${ }^{1}$ Radiology Department, Hospital Naval Almirante Nef, Subida Alesandri S/N., Viña del Mar, Provincia de Valparaíso, Chile. ${ }^{2}$ Radiology Department, Hospital Dr. Eduardo Pereira, Valparaiso, Chile. ${ }^{3}$ Intensive Care Unit, Hospital Naval Almirante Nef, Viña del Mar, Chile. ${ }^{4}$ Radiology Department, Clinica Bupa Santiago, Santiago, Chile. ${ }^{5}$ Intensive Care Unit, Hospital San Martin de Quillota, Quillota, Chile. ${ }^{6}$ Intensive Care Unit, Hospital IESS Los Ceibos, Guayaquil, Ecuador. ${ }^{7}$ Radiology Department, Centro de Diagnóstico Dr. Enrique Rossi, Buenos Aires, Argentina. ${ }^{8}$ Viña del Mar, Escuela de Medicina, Facultad de Medicina Viña del Mar, Universidad Andres Bello, Valparaiso, Chile.

Received: 24 July 2020 Accepted: 6 October 2020 Published online: 21 October 2020

\section{References}

1. COVID-19 Dashboard by the Center for Systems Science and Engineering (CSSE) at Johns Hopkins University (JHU). https://coronavirus.jhu.edu/ map.html. Accessed 22 July 2020.

2. Wu Z, McGoogan JM. Characteristics of and important lessons from the coronavirus disease 2019 (COVID-19) outbreak in China: summary of a report of 72314 cases from the Chinese Center for Disease Control and Prevention. JAMA. 2020;323(13):1239-42. https://doi.org/10.1001/ jama.2020.2648.

3. Gattinoni L, Chiumello D, Rossi S. COVID-19 pneumonia: ARDS or not? Crit Care. 2020;24:154. https://doi.org/10.1186/s13054-020-02880-z.

4. Lang M, Som A, Mendoza DP, Flores EJ, Reid N, Carey D, Li MD, Witkin A, Rodriguez-Lopez JM, Shepard JA, Little BP. Hypoxaemia related to COVID19: vascular and perfusion abnormalities on dual-energy CT. Lancet Infect Dis. 2020. https://doi.org/10.1016/S1473-3099(20)30367-4.

5. Oudkerk M, Büller HR, Kuijpers D, van Es N, Oudkerk SF, McLoud TC, Gommers D, van Dissel J, Ten Cate H, van Beek EJ. Diagnosis, prevention, and treatment of thromboembolic complications in COVID-19: report of the National Institute for Public Health of the Netherlands. Radiology. 2020. https://doi.org/10.1148/radiol.2020201629.

6. Thachil J, Agarwal S. Understanding the COVID-19 coagulopathy spectrum. Anaesthesia. 2020. https://doi.org/10.1111/anae.15141.

7. Gris JC, Perez-Martin A, Quéré I, Sotto A. COVID-19 associated coagulopathy: the crowning glory of thrombo-inflammation concept. Anaesth Crit Care Pain Med. 2020. https://doi.org/10.1016/j.accpm.2020.04.013 (Epub ahead of print).

8. Engelmann B, Massberg S. Thrombosis as an intravascular effector of innate immunity. Nat Rev Immunol. 2013;13(1):34-45. https://doi. org/10.1038/nri3345.

9. Varga Z, Flammer AJ, Steiger P, Haberecker M, Andermatt R, Zinkernagel AS, Mehra MR, Schuepbach RA, Ruschitzka F, Moch H. Endothelial cell infection and endotheliitis in COVID-19. Lancet. 2020;395(10234):1417-8. https://doi.org/10.1016/S0140-6736(20)30937-5.

10. Gheblawi M, Wang K, Viveiros A, Nguyen Q, Zhong JC, Turner AJ, Raizada MK, Grant MB, Oudit GY. Angiotensin-converting enzyme 2: SARS-CoV-2 receptor and regulator of the renin-angiotensin system: celebrating the 20th anniversary of the discovery of ACE2. Circ Res. 2020;126(10):145674. https://doi.org/10.1161/CIRCRESAHA.120.317015.

11. Liu Y, Yang Y, Zhang C, Huang F, Wang F, Yuan J, et al. Clinical and biochemical indexes from 2019-nCoV infected patients linked to viral loads and lung injury. Sci China Life Sci. 2020;63(3):364-74. https://doi. org/10.1007/s11427-020-1643-8.

12. Wu Z, Hu R, Zhang C, Ren W, Yu A, Zhou X. Elevation of plasma angiotensin II level is a potential pathogenesis for the critically ill COVID-19 patients. Crit Care. 2020;24:290. https://doi.org/10.1186/s13054-02003015-0.

13. Liu P, Blet A, Smyth D, Li H. The science underlying COVID-19: implications for the cardiovascular system. Circulation. 2020. https://doi.org/10.1161/ CIRCULATIONAHA.120.047549.

14. Wang K, Gheblawi M, Oudit G. Angiotensin converting enzyme 2: a double-edged sword. Circulation. 2020. https://doi.org/10.1161/CIRCU LATIONAHA.120.047049.

15. Hedenstierna G, Chen L, Hedenstierna M, Scaramuzzo G. Treatment of COVID-19 by inhaled NO to reduce shunt? Am J Respir Crit Care Med. 2020. https://doi.org/10.1164/rccm.202004-0940LE.

16. Coppo A, Bellani G, Winterton D, Di Pierro M, Soria A, Faverio P, Cairo M, Mori S, Messinesi G, Contro E, Bonfanti P. Feasibility and physiological effects of prone positioning in non-intubated patients with acute respiratory failure due to COVID-19 (PRON-COVID): a prospective cohort study. Lancet Respir Med. 2020. https://doi.org/10.1016/S2213-2600(20)30268 $-X$.

17. Zhang $L J$, Zhou CS, Schoepf UJ, Sheng HX, Wu SY, Krazinski AW, Silverman JR, Meinel FG, Zhao YE, Zhang ZJ, Lu GM. Dual-energy CT lung ventilation/perfusion imaging for diagnosing pulmonary embolism. Eur Radiol. 2013;23(10):2666-75. https://doi.org/10.1259/bjr/16337436.

18. Lu GM, Zhao YE, Zhang LJ, Schoepf UJ. Dual-energy CT of the lung. Am J Roentgenol. 2012;199(5_supplement):S40-53. https://doi.org/10.2214/ AJR.12.9112.

19. Tamura M, Yamada Y, Kawakami T, Kataoka M, Iwabuchi Y, Sugiura H, Hashimoto M, Nakahara T, Okuda S, Nakatsuka S, Sano F. Diagnostic accuracy of lung subtraction iodine mapping CT for the evaluation of pulmonary perfusion in patients with chronic thromboembolic pulmonary hypertension: correlation with perfusion SPECT/CT. Int J Cardiol. 2017;243:538-43. https://doi.org/10.1016/j.ijcard.2017.05.006.

20. Grob D, Smit E, Prince J, Kist J, Stöger L, Geurts B, Snoeren MM, van Dijk R, Oostveen LJ, Prokop M, Schaefer-Prokop CM. Iodine maps from subtraction CT or dual-energy CT to detect pulmonary emboli with CT 
angiography: a multiple-observer study. Radiology. 2019;292(1):197-205. https://doi.org/10.1148/radiol.2019182666.

21. Dissaux B, Le Floch PY, Robin P, Bourhis D, Couturaud F, Salaun PY, Nonent $M$, Le Roux PY. Pulmonary perfusion by iodine subtraction maps $C T$ angiography in acute pulmonary embolism: comparison with pulmonary perfusion SPECT (PASEP trial). Eur Radiol. 2020;30:4857-64. https://doi org/10.1007/s00330-020-06836-3.

\section{Publisher's Note}

Springer Nature remains neutral with regard to jurisdictional claims in published maps and institutional affiliations.
Ready to submit your research? Choose BMC and benefit from:

- fast, convenient online submission

- thorough peer review by experienced researchers in your field

- rapid publication on acceptance

- support for research data, including large and complex data types

- gold Open Access which fosters wider collaboration and increased citations

- maximum visibility for your research: over 100M website views per year

At BMC, research is always in progress.

Learn more biomedcentral.com/submissions 